\title{
AG490 reverses phenotypic alteration of dendritic cells by bladder cancer cells
}

\author{
WEIGANG XIU ${ }^{1,2}$, JUAN MA ${ }^{1,2}$, TING LEI ${ }^{1,2}$ and MAN ZHANG ${ }^{1-3}$ \\ ${ }^{1}$ Department of Clinical Laboratory Medicine, Beijing Shijitan Hospital, Capital Medical University; \\ ${ }^{2}$ Beijing Key Laboratory of Urinary Cellular Molecular Diagnostics; ${ }^{3}$ Department of Clinical Laboratory Medicine, \\ Peking University Ninth School of Clinical Medicine, Beijing 100038, P.R. China
}

Received March 7, 2017; Accepted April 18, 2018

DOI: $10.3892 / \mathrm{ol} .2018 .9028$

\begin{abstract}
Past studies have confirmed that tumors can impair the function of dendritic cells (DCs) and promote tumor evasion. AG490, a Janus kinase 2/signal transducer and activator of transcription 3 inhibitor, has been shown to induce maturation of DCs and inhibit the growth of tumor cells. In the present study, DCs were generated from healthy human peripheral blood mononuclear cells. On day 5 of culture, the DCs were co-cultured with human bladder cancer pumc-91 cells for $24 \mathrm{~h}$, and then purified using magnetic beads. The maturation of the DCs was induced by lipopolysaccharide. Subsequent to co-culture with pumc-91 cells, the expression of human leukocyte antigen-antigen D related (HLA-DR), cluster of differentiation (CD)86 and CD80 was found to be reduced in the DCs, accompanied by increased production of interleukin (IL)-10, but decreased production of IL-12p70. Furthermore, the DCs co-cultured with pumc-91 inhibited the proliferation of allogeneic T cells. Finally, AG490 restored the expression of HLA-DR, CD86 and CD80. These data identified that bladder cancer cells could inhibit the antigen-presenting function of the DCs and induce anergy in T cells. AG490 may partly reverse this inhibitory effect of bladder cancer cells on DCs, activate immunogenicity and induce the antitumor immunity response of DCs.
\end{abstract}

\section{Introduction}

Bladder cancer is one of the most common malignancies in the urinary system. The biological characteristics of bladder cancer are recurrence and drug resistance (1). While 50-70\% of non-muscle invasive bladder cancer (NMIBC) patients experience recurrence, $50-80 \%$ of MIBC patients develop

Correspondence to: Dr Man Zhang, Department of Clinical Laboratory Medicine, Beijing Shijitan Hospital, Capital Medical University, 10 Tieyi Road, Haidian, Beijing 100038, P.R. China E-mail: zhangman@bjsjth.cn

Key words: bladder cancer cell, dendritic cell, AG490, Janus kinase 2/signal transducer and activator of transcription 3 pathway progression to distant metastasis (2). A transurethral resection of the bladder tumor (TURBT) is the primary method of treating NMIBC patients. TURBT is also an important diagnostic and staging tool in the management of bladder cancer patients. Radical cystectomy and pelvic lymph node dissection provide accurate evaluation of MIBC patients, and this evaluation could avoid errors in clinical staging $(3,4)$. Meanwhile, the results of animal studies and clinical trials have suggested that dendritic cell (DC)-based immunotherapy may have the potential for activating immune responses and for a therapeutic effect in bladder cancer (5).

DCs are key cells in the initiation of innate and adaptive immunity, with have the ability to modulate the antitumor immune response (6). DCs are the most powerful antigen-presenting cells and only DCs have the ability for activation of the primary sensitization against specific antigens in a naïve host (7). However, recent findings have shown that DCs will be dysfunctional in the tumor microenvironment (8). Tumor-derived factors affect DC maturation and differentiation. Immature DCs (iDCs) fail to supply costimulatory molecules to T cells and antitumor immune tolerance or anergy may develop. Moreover, tumor-infiltrating DCs (TIDCs) have been demonstrated to be associated with low expression of costimulatory signal, ineffective antigen cross-presentation, and high expression of regulatory molecules and receptors (9).

Constitutive activation of the signal transducer and activator of transcription 3 (STAT3) signaling pathway serves a pivotal role in the growth and invasion of bladder cancer (10). AG490 is a Janus kinase 2 (Jak2)/STAT3 signaling pathway inhibitor, which reverses the inhibitory effect of hyperphosphorylated STAT3 on DC differentiation (11). Moreover, AG490 also inhibits vascular endothelial growth factor expression by control over the downstream target genes of the Jak2/STAT3 pathway. Therefore, AG490 could provide a basis for targeting anti-angiogenesis and exhibit therapeutic significance in the treatment of bladder cancer patients (10).

The purpose of the present study was to investigate the associations between bladder cancer cells and DCs. Human bladder cancer pumc-91 cells co-cultured with human peripheral blood mononuclear cell (PMBC)-derived DCs were used for the DC surface phenotyping assays, cytokine production assays and allogeneic mixed leukocyte reaction assay. Furthermore, the study also investigated how AG490 
may reverse the inhibitory effects of bladder cancer cells on the phenotype of DCs.

\section{Materials and methods}

Antibodies. The following antibodies were supplied in PBS and used without further dilution in the study: Phycoerythrin-labeled, anti-cluster of differentiation (CD) 86 (cat no. 12-0869-42), anti-CD80 (cat no. 12-0809-42), anti-human leukocyte antigen (HLA) class I molecules HLA-A, HLA-B and HLA-C antigens (HLA-ABC; cat no. 12-9983-42), anti-HLA-antigen D related (HLA-DR; cat no. 17-9956-42), PE isotype control mAbs (cat no. 12-4724-81), APC isotype control mAbs (cat no. 17-4714-81), Mouse IgG (cat no. 25-4714-42) and allophycocyanin-labeled anti-CD11c (cat no. 17-0116-42) (all eBioscience; Thermo Fisher Scientific, Inc., Waltham, MA, USA).

Cell culture. The human bladder cancer pumc-91 cell line was provided by Peking Union Medical College Hospital (Beijing, China) (12). Pumc-91 cells were cultured in RPMI-1640 medium (Gibco; Thermo Fisher Scientific, Inc.) supplemented with $10 \%$ heat-inactivated fetal bovine serum (FBS; Ausbian, Sydney, Australia) at $37^{\circ} \mathrm{C}$ and $5 \% \mathrm{CO}_{2}$. Human PBMCs from healthy volunteers were isolated immediately from $100 \mathrm{ml}$ buffy coat according to standard protocols using Ficoll-Hypaque density gradient centrifugation at $700 \mathrm{x} \mathrm{g}$ for $30 \mathrm{~min}$ at $20^{\circ} \mathrm{C}$. To generate DCs, PBMCs were cultured at a density of $5 \times 10^{6}$ cells $/ 2 \mathrm{ml}$ in a 6 -well plate (Greiner Bio-One, Kremsmünster, Austria) in AIM-V medium (Gibco; Thermo Fisher Scientific, Inc.) containing $10 \%$ FBS. The plate was incubated at $37^{\circ} \mathrm{C}$ for $2 \mathrm{~h}$, and the non-adherent cells were discarded. The adherent cells were treated for 6 days at $37^{\circ} \mathrm{C}$ in complete AIM-V medium supplemented with $10 \% \mathrm{FBS}$, $50 \mathrm{ng} / \mathrm{ml}$ recombinant human (rh)-granulocyte-macrophage colony-stimulating factor and $50 \mathrm{ng} / \mathrm{ml} \mathrm{rh-interleukin-4}$ (rhIL-4) (Peprotech, Inc., Rocky Hill, NJ, USA) to generate iDCs. Half-volume medium replacement with all cytokines was performed on days 3 and 5. The samples were collected on August 21, 2015, in Beijing Shijitan Hospital, Capital Medicine University (Beijing, China). The study was approved by the Ethics Board of Beijing Shijitan Hospital, Capital Medicine University (Beijing, China).

Bladder cancer cell treatment with AG490. AG490 was dissolved in dimethyl sulfoxide (DMSO; both Sigma-Aldrich; Merck KGaA, Darmstadt, Germany) and diluted to a final concentration of $200 \mu \mathrm{M}$. Pumc-91 cells were treated for $24 \mathrm{~h}$ with AG490 at $200 \mu \mathrm{M}$. Subsequently, AG490-treated pumc-91 (pumc-91/AG490) cells were washed three times and co-cultured with DCs at $37^{\circ} \mathrm{C}$ for 24 h. $1.2 \%$ DMSO-treated pumc-91 (pumc-91/DMSO) cells were used as the control group.

DC co-culture with bladder cancer cells. On day 5 of DC culture, $1 \times 10^{6}$ pumc-91 or pumc-91/AG490 cells were added to each well containing the DCs. On day 6, these cells were washed three times and purified with microbeads from a QuadroMACS Starting kit (LD columns) and MiniMACS Starting kit (MS columns) using a Blood Dendritic
Isolation kit II (cat no. 130-091-379, MiltenyiBiotec GmbH, BergischGladbach, Germany). Non-DC Depletion Cocktail and DC Enrichment Cocktail are contained within the Blood Dendritic Cell Isolation kit II. The following antibodies were not pre-diluted and were dissolved in running buffer at the ratio of 1:3. (cat. no. 130-091-221; MiltenyiBiotec $\mathrm{GmbH}$, Bergisch Gladbach, Germany). Non-DC Depletion Cocktail includes monoclonal biotin-conjugated antibodies against human CD1c, CD14 and CD19. DC Enrichment Cocktail includes microbeads conjugated to monoclonal antibodies against human CD304, CD141 and biotin. For iDC activation, $1 \mu \mathrm{g} / \mathrm{ml}$ LPS (Sigma-Aldrich; Merck KGaA) was added at day 6 and the culture was continued for $24 \mathrm{~h}$ to induce mature DCs (mDCs).

DC surface phenotyping. The phenotype of the DCs was analyzed by flow cytometry. The DCs were harvested and stained with antibodies. DCs were blocked by mouse IgG $\kappa$ (cat no. 25-4714-42; eBioscience; Thermo Fisher Scientific, Inc.) at $4^{\circ} \mathrm{C}$ for $30 \mathrm{~min}$, then stained with a PE-labeled, APC-labeled specific $\mathrm{mAb}$ or isotype control to detect the target molecule at $4^{\circ} \mathrm{C}$ for $30 \mathrm{~min}$. Mouse $\operatorname{IgG}(5 \mu \mathrm{l})$ were supplied in PBS for each sample and used without further dilution. DCs were stained with specific monoclonal antibodies, PE isotype control mAbs or APC isotype control mAbs (5 $\mu \mathrm{l})$ (as previously described) at $4^{\circ} \mathrm{C}$ for $30 \mathrm{~min}$, and then washed twice and resuspended in phosphate-buffered saline. Stained cells were analyzed by a flow cytometer (CytoFLEX; Beckman Coulter, Inc., Brea, CA, USA). Data of mean fluorescence intensity and percentage of positive cells were acquired and processed using accompanying software (CytExpert 1.0; Beckman Coulter, Inc.).

Cytokine production assays. Purified mDCs were cultured in a 6 -well plate at $1.8 \times 10^{5}$ cells per well. Human ELISA Ready-SET-Go kits (eBioscience; Thermo Fisher Scientific, Inc., Waltham, MA, USA) were used according to the manufacturer's protocols in order to measure the levels of IL-10 (cat. no. 88-7106) and IL-12p70 (cat. no. 88-7126).

Allogeneic mixed leukocyte reaction assay. PBMCs were obtained from healthy human peripheral blood and isolated by Ficoll-Hypaque density gradient centrifugation, as aforementioned. Labeled $\mathrm{CD}^{+} \mathrm{T}$ cells were purified from the PBMCs using the Pan $\mathrm{T}$ cell Isolation kit (MiltenyiBiotec $\mathrm{GmbH}$ ). In a previous experiment, it was found that $\mathrm{DC} / \mathrm{T}$ cells at the ratio of 1:20 showed the best result in terms of $\mathrm{T}$ cell proliferation according to the OD value (13). The purified $\mathrm{CD}^{+} \mathrm{T}$ cells were seeded into a round-bottom 96 -well plate at $2.0 \times 10^{5}$ cells per well. The purified DCs were irradiated (30 Gy for $30 \mathrm{~min}$ ), incubated with allogeneic $\mathrm{CD}^{+} \mathrm{T}$ cells at $\mathrm{DC} / \mathrm{T}$ cell ratios of 1:20 and cultured for 5 days with Cell Counting kit- 8 (Dojindo Molecular Technologies, Inc., Kumamoto, Japan) for $4 \mathrm{~h}$.

Statistical analysis. Data are presented as the mean \pm standard deviation (SD). All the experiments were repeated three times. The statistical analysis was performed using a one-way analysis of variance (ANOVA) and least significant difference method with SPSS 17.0 statistical software (SPSS Inc., Chicago, IL, USA). $\mathrm{P}<0.05$ was considered to indicate a statistically significant difference. 


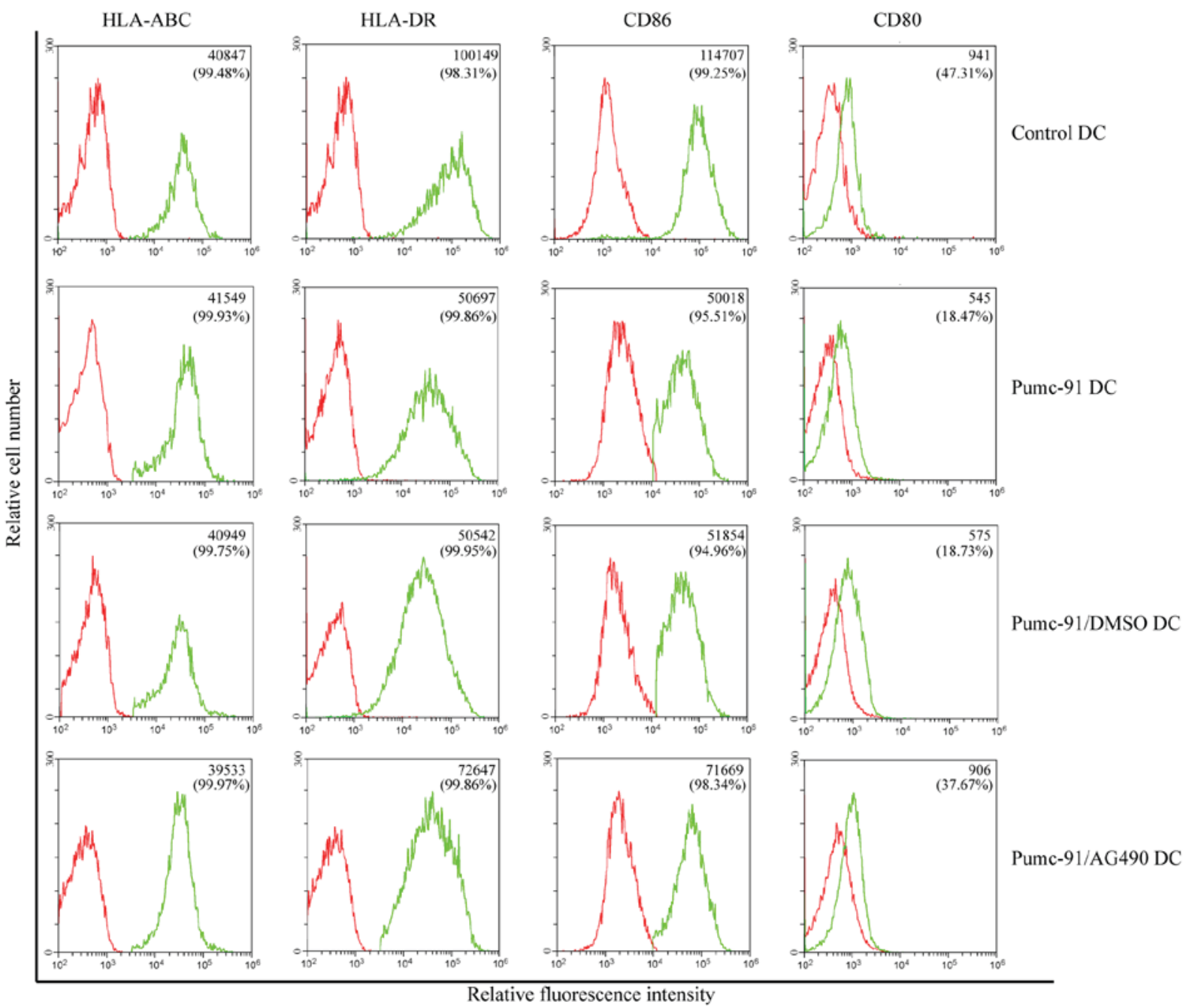

Figure 1. Effects of bladder cancer cells on DC phenotype. DCs were co-cultured with pumc-91 cells or AG490-treated pumc-91 cells for $24 \mathrm{~h}$, and then maturation was induced with lipopolysaccharide. The expression of DC surface markers was tested by flow cytometery. The mean fluorescence intensity and percentage of positive cells are presented in the top right corner of each panel. Data shown are representative of three experiments. DCs, dendritic cells; DMSO, dimethyl sulfoxide; HLA-DR, human leukocyte antigen-antigen D related; HLA-ABC, HLA class I molecules HLA-A, HLA-B and HLA-C antigens; $\mathrm{CD}$, cluster of differentiation.

\section{Results}

Inhibition of DC phenotype by co-culture with bladder cancer cells. First, the effect of bladder cancer cells on DC phenotype was assessed. On day 7, the expression of major histocompatibility complex (MHC) class molecules (HLA-ABC and HLA-DR) and costimulatory molecules (CD86 and CD80) was determined. Compared with control DCs, pumc-91 cells co-cultured with DCs exhibited reduced expression of HLA-DR, CD86 and CD80. However, there were no differences between pumc-91-exposed DCs and DCs co-cultured with pumc-91/DMSO. These observations were found based on mean fluorescence intensity (MFI) results and the percentage of positive cells (Fig. 1). The DCs in the three tests were obtained from two donors, but there were no individual differences. The statistical analysis of MFI was calculated by one-way ANOVA. Furthermore, the purity of the DC samples was shown to be $>93 \%$ in all experiments (Fig. 2).

Bladder cancer cells inhibit IL-12p70 production while increasing $I L-10$ secretion of DCs. In addition to MHC class molecules and costimulatory molecules, cytokines secreted from DCs serve an important role in priming the cytotoxic lymphocyte response. IL-10 and IL-12p70 were detected in supernatants of control DCs and DCs co-cultured with pumc-91 cells. Upon LPS stimulation, the pumc-91 co-cultured with DCs produced a marked increase in IL-10 level, while IL-12p70 level was decreased, compared with that in the control DCs $(\mathrm{P}<0.05)$ (Fig. 3).

Inefficient T cell stimulation by DCs co-cultured with pumc-91. Next, the immunostimulatory effect of pumc-91-exposed DCs was tested. PBMC-derived DCs generated in the presence or absence of pumc-91 were assessed by measuring their ability to induce the proliferation of allogeneic $\mathrm{CD}^{+} \mathrm{T}$ cells. Compared with control DCs, DCs co-cultured with pumc-91 cells showed significantly less potency in stimulating allogeneic $\mathrm{CD}^{+}$ T cell proliferation (Fig. 4). Therefore, bladder cancer pumc-91 cells affected the maturation of the DCs along with providing an inhibitory effect on the function of the DCs.

AG490 reverses the inhibitory effect of bladder cancer cells on DC phenotype. PBMC-derived DCs were co-cultured with 


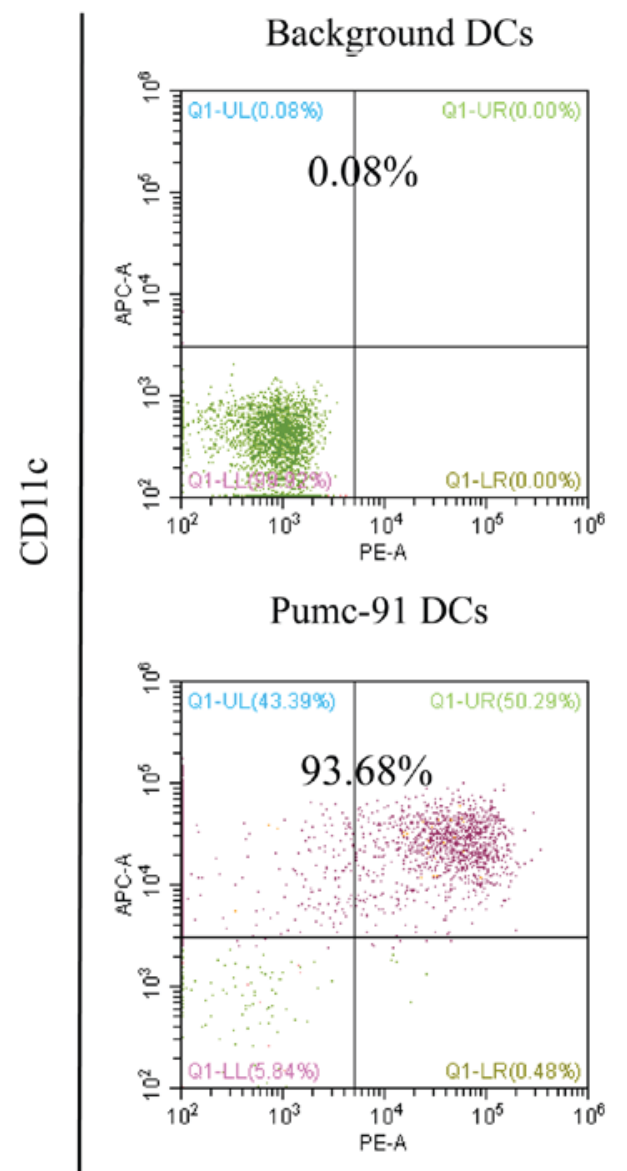

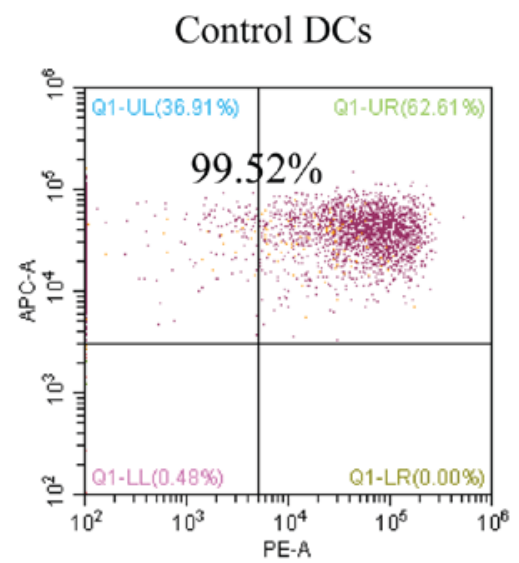

Pumc-91/AG490 DCs

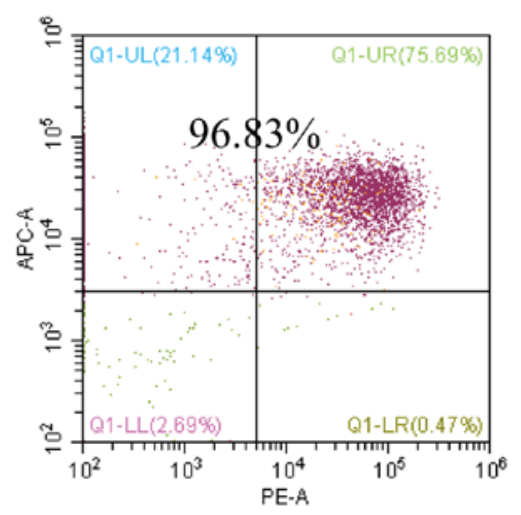

\section{CD86}

Figure 2. Percentage of purified DC samples in all experiments. DCs were labeled with the Non-DC Depletion Cocktail for negative selection. Next, DCs were labeled with DC Enrichment Cocktail for positive selection. The expression of CD11c and CD86 was tested by flow cytometery. The percentages of purified DC are presented in the top of each panel. DCs, dendritic cells; CD, cluster of differentiation.

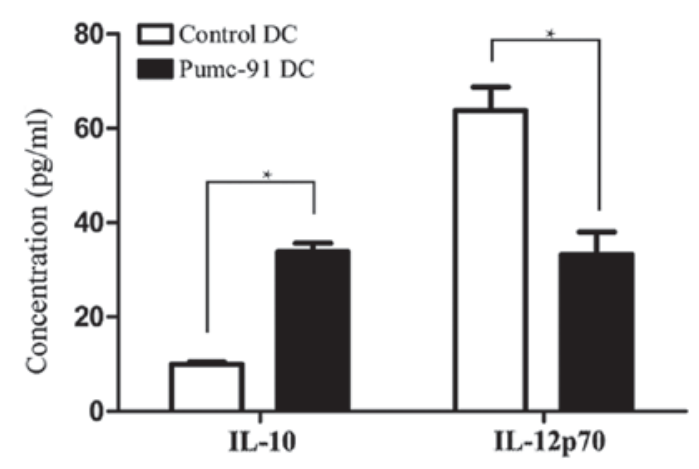

Figure 3. Production of IL-10 is increased, but production of IL-12p70 is decreased in DCs co-cultured with bladder cancer pumc-91 cells. Control DCs or pumc-91-co-cultured DCs were treated with lipopolysaccharide for $24 \mathrm{~h}$, and the levels of IL-10 and IL-12p70 in cell-free supernatants were measured by ELISA. Values shown are the mean \pm standard deviation from triplicate representative experiments. " $\mathrm{P}<0.05$. DCs, dendritic cells; IL, interleukin.

pumc-91 cells for $24 \mathrm{~h}$ with or without pretreatment with AG490. Compared with the DCs co-cultured with pumc-91 cells, the DCs exposed to AG490-treated pumc-91 cells exhibited upregulated expression of HLA-DR, CD86 and CD80 (Fig. 1). This revealed that blocking pumc-91-induced STAT3 activation by pretreatment with AG490 reversed the inhibitory effect of the pumc-91 cells on the DC phenotype.

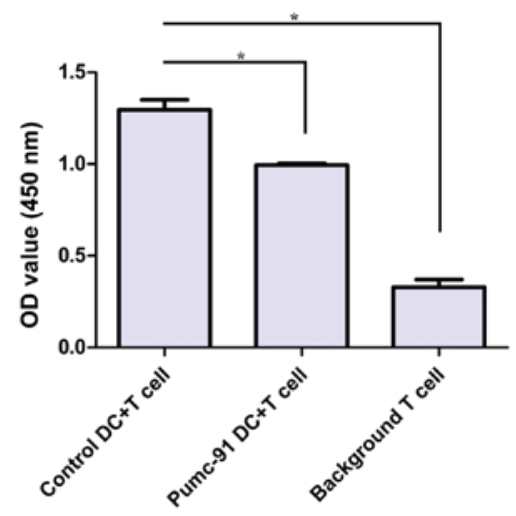

Figure 4. Effects of bladder cancer pumc-91 cells on DC allostimulatory function. Control DCs or pumc-91-exposed DCs were treated with LPS for $24 \mathrm{~h}$. The LPS-matured DCs were irradiated and incubated with allogeneic cluster of differentiation $3^{+} \mathrm{T}$ cells at a $\mathrm{DC} / \mathrm{T}$ cell ratio of 1:20 for 5 days. The proliferation of T cells was measured at $450 \mathrm{~nm}$ by Cell Counting kit- 8 . Values shown are the mean \pm standard deviation from triplicate representative experiments. " $\mathrm{P}<0.05$. DCs, dendritic cells; LPS, lipopolysaccharide; OD, optical density.

\section{Discussion}

The results of the present study indicate that bladder cancer cells may evade the host defense response by inhibiting DC 
maturation, and by inducing IL-10 secretion and suppressing IL-12p70 production in DCs. It was also found that bladder cancer cell co-cultured DCs failed to develop a full allostimulatory ability for $\mathrm{CD}^{+} \mathrm{T}$ lymphocytes. In addition, blocking bladder cancer cell-induced STAT3 activation by AG490 effectively reversed the inhibitory effect on DC maturation.

DCs are known as the most powerful antigen presenting cells (14). Previous studies have suggested that the immunostimulatory ability of DCs could be conditioned by a number of factors present in the tumor microenvironment $(13,15,16)$. Tolerogenic properties of DCs are usually attributed to their immature status (17). In the present study, PBMC-derived DCs co-cultured with pumc-91 cells for $24 \mathrm{~h}$ exhibited an immature phenotype. Compared with control DCs, DCs co-cultured with pumc-91 exhibited low expression of HLA-DR, CD86 and CD80. This may partly explain why TIDCs show a phenotype of low costimulatory molecule expression, and DCs may be polarized into immunosuppressive regulatory DCs (18-20).

In addition to the immature phenotype in tumor lesions, the same finding was obtained in the peripheral blood of patients with tumors. This result suggests that tumor-associated soluble factors may prevent DC function (9). One of the cytokines reported to suppress the function of DCs is IL-10. IL-10 inhibits the capacity of DCs to stimulate the proliferation of T lymphocytes (21). Overexpression of IL-10 in DCs could suppress the allogeneic cytotoxic lymphocyte response and IL-12p70 production (22). This is consistent with the present study findings. It was found that pumc-91 cells co-cultured with DCs produced a lower level of IL-12p70, but a higher level of IL-10 compared with controls. The decreased level of IL-12p70 may prevent the development of the type $1 \mathrm{~T}$ helper response, and result in a lack of IFN- $\gamma$ supporting the cytotoxic lymphocyte response $(23,24)$.

It has been well established that the interaction of CD86 and CD80 with CD28 receptor results in the activation of costimulatory signals of DCs (25). These molecules are indispensable to prime the $\mathrm{T}$ cytotoxic lymphocyte response. Therefore, the inhibition of CD86 and CD80 by pumc-91 cells may suggest that the bladder cancer cells disrupted the antigen-specific immune-activating abilities of the DCs to induce a T cell response. Moreover, IL-10 could promote the apoptosis of DCs and reverse the protection against apoptosis conferred by tumor necrosis factor- $\alpha$ and CD40 ligand (26). The apoptosis of DCs may reduce the time window during which they contact with $\mathrm{T}$ lymphocytes. Consistent with these findings, the present observed that pumc-91 cell-exposed DCs could not stimulate proliferation of allogeneic $\mathrm{CD}^{+} \mathrm{T}$ cells. This also suggests that tumor-associated soluble factors could impair the antigen-presenting function of DCs.

Constitutive activation of the STAT3 signal pathway has been observed in several types of tumors, and it could promote the proliferation and invasion of tumor cells (27). AG490 is an effective inhibitor of the Jak2/STAT3 pathway. A previous study showed that prior treatment with AG490 could reverse human pancreatic cancer cell conditioned medium-induced inhibition of DC differentiation (11). Moreover, AG490 could induce tumor apoptosis and promote DC maturation. AG490 did not have any side effect on activity and function. Therefore, it is suggested that AG490 could act as an adjuvant used in DC-based immunotherapy (28). In the present study, compared with pumc-91 co-cultured DCs, AG490-treated pumc-91-exposed DCs exhibited upregulation of CD86, CD80 and HLA-DR. This may suggest that pretreatment with AG490 could inhibit STAT3 activation in bladder cancer cells and reverse the inhibitory effect of pumc-91 cells on the DC phenotype. Furthermore, Jak2/STAT3 may serve a vital role in inducing the immunosuppressive effect of bladder cancer on the function of DCs.

In conclusion, the present study found that bladder cancer pumc-91 cells could inhibit DC maturation and weaken their ability to activate $\mathrm{T}$ cells. It is possible that this is one of the reasons for DC-based therapies yielding unsatisfactory therapeutic effects. Finally, the use of AG490 was demonstrated to partly reverse DC dysfunction.

\section{Acknowledgements}

Not applicable.

\section{Funding}

The present study was supported by the Beijing Natural Science Foundation (grant no. 7172106).

\section{Availability of data and materials}

The datasets used and/or analyzed during the current study are available from the corresponding author on reasonable request.

\section{Authors' contributions}

MZ designed the study. WX, JM and TL performed the experiments. WX wrote the paper. JM, TL and MZ reviewed and edited the manuscript. All authors read and approved the manuscript.

\section{Ethics approval and consent to participate}

The study was approved by the Ethics Board of Beijing Shijitan Hospital, Capital Medicine University (Beijing, China). All volunteers provided written informed consent.

\section{Patient consent for publication}

The volunteers provided consent for publication.

\section{Competing interests}

The authors declare that they have no competing interests.

\section{References}

1. Lei T, Zhao X, Jin S, Meng Q, Zhou H and Zhang M: Discovery of potential bladder cancer biomarkers by comparative urine proteomics and analysis. Clin Genitourin Cancer 11: 56-62, 2013.

2. Meng Q, Lei T, Zhang M, Zhao J, Zhao XH and Zhang M: Identification of proteins differentially expressed in adriamycin-resistant (pumc-91/ADM) and parental (pumc-91) human bladder cancer cell lines by proteome analysis. J Cancer Res Clin Oncol 139: 509-519, 2013. 
3. Stein JP, Lieskovsky G, Cote R, Groshen S, Feng AC, Boyd S, Skinner E, Bochner B, Thangathurai D, Mikhail M, et al: Radical cystectomy in the treatment of invasive bladder cancer: Long-term results in 1,054 patients. J Clin Oncol 19: 666-675, 2001 .

4. Amling CL, Thrasher JB, Frazier HA, Dodge RK, Robertson JE and Paulson DF: Radical cystectomy for stages Ta, Tis and T1 transitional cell carcinoma of the bladder. J Urol 151: 31-35; discussion 35-36, 1994

5. Schuler G, Schuler-Thurner B and Steinman RM: The use of dendritic cells in cancer immunotherapy. Curr Opin Immunol 15: $138-147,2003$

6. Si C, Zhang R, Wu T, Lu G, Hu Y, Zhang H, Xu F, Wei P, Chen K, Tang H, et al: Dendritic cell-derived nitric oxide inhibits the differentiation of effector dendritic cells. Oncotarget 7: 74834-74845, 2016.

7. Ma J, Usui Y, Takeuchi M, Okunuki Y, Kezuka T, Zhang L, Mizota A and Goto H: Human uveal melanoma cells inhibit the immunostimulatory function of dendritic cells. Exp Eye Res 91: 491-499, 2010.

8. Ma Y, Shurin GV, Peiyuan Z and Shurin MR: Dendritic cells in the cancer microenvironment. J Cancer 4: 36-44, 2013.

9. Pinzon-Charry A, Maxwell T and López JA: Dendritic cell dysfunction in cancer: A mechanism for immunosuppression. Immunol Cell Biol 83: 451-461, 2005.

10. Joung YH, Na YM, Yoo YB, Darvin P, Sp N, Kang DY, Kim SY, Kim HS, Choi YH, Lee HK, et al: Combination of AG490, a Jak2 inhibitor, and methylsulfonylmethane synergistically suppresses bladder tumor growth via the Jak2/STAT3 pathway. Int J Oncol 44: 883-895, 2014.

11. Bharadwaj U, Li M, Zhang R, Chen C and Yao Q: Elevated interleukin-6 and G-CSF in human pancreatic cancer cell conditioned medium suppress dendritic cell differentiation and activation. Cancer Res 67: 5479-5488, 2007.

12. Yu S, Meng Q, Hu H and Zhang M: Correlation of ANXA1 expression with drug resistance and relapse in bladder cancer. Int J Clin Exp Pathol 7: 5538-5548, 2014.

13. Xiu W, Ma J, Lei T, Zhang M and Zhou S: Immunosuppressive effect of bladder cancer on function of dendritic cells involving of Jak2/STAT3 pathway. Oncotarget 7: 63204-63214, 2016.

14. Goyvaerts $\mathrm{C}$ and Breckpot K: Pros and cons of antigen-presenting cell targeted tumor vaccines. J Immunol Res 2015: 785634, 2015 .

15. Zhang M, Tang H, Guo Z, An H, Zhu X, Song W, Guo J, Huang X, Chen T, Wang J and Cao X: Splenic stroma drives mature dendritic cells to differentiate into regulatory dendritic cells. Nat Immunol 5: 1124-1133, 2004.

16. Svensson M, Maroof A, Ato M and Kaye PM: Stromal cells direct local differentiation of regulatory dendritic cells. Immunity 21 : 805-816, 2004
17. Lizée G, Radvanyi LG, Overwijk WW and Hwu P: Improving antitumor immune responses by circumventing immunoregulatory cells and mechanisms. Clin Cancer Res 12: 4794-4803, 2006.

18. Tran Janco JM, Lamichhane P, Karyampudi L and Knutson KL: Tumor-infiltrating dendritic cells in cancer pathogenesis. J Immunol 194: 2985-2991, 2015.

19. Harimoto $H$, Shimizu M, Nakagawa $Y$, Nakatsuka $K$, Wakabayashi A, Sakamoto $\mathrm{C}$ and Takahashi H: Inactivation of tumor-specific $\mathrm{CD}^{+}$CTLs by tumor-infiltrating tolerogenic dendritic cells. Immunol Cell Biol 91: 545-555, 2013.

20. Krempski J, Karyampudi L, Behrens MD, Erskine CL, Hartmann L, Dong H, Goode EL, Kalli KR and Knutson KL: Tumor-infiltrating programmed death receptor- $1^{+}$dendritic cells mediate immune suppression in ovarian cancer. J Immunol 186 6905-6913, 2011.

21. Steinbrink K, Jonuleit H, Muller G, Schuler G, Knop J and Enk AH: Interleukin-10-treated human dendritic cells induce a melanoma-antigen-specific anergy in CD8(+) T cells resulting in a failure to lyse tumor cells. Blood 93: 1634-1642, 1999.

22. Steinbrink K, Graulich E, Kubsch S, Knop J and Enk AH: CD4(+) and CD8(+) anergic T cells induced by interleukin-10-treated human dendritic cells display antigen-specific suppressor activity. Blood 99: 2468-2476, 2002.

23. Hilkens CM, Kalinski P, de Boer M and Kapsenberg ML: Human dendritic cells require exogenous interleukin-12-inducing factors to direct the development of naive T-helper cells toward the Th1 phenotype. Blood 90: 1920-1926, 1997.

24. Jackson AM, Mulcahy LA, Zhu XW, O'Donnell D and Patel PM: Tumour-mediated disruption of dendritic cell function: Inhibiting the MEK1/2-p44/42 axis restores IL-12 production and Th1-generation. Int J Cancer 123: 623-632, 2008.

25. Collins M, Ling V and Carreno BM: The B7 family of immune-regulatory ligands. Genome Biol 6: 223, 2005.

26. Ludewig B, Graf D, Gelderblom HR, Becker Y, Kroczek RA and Pauli G: Spontaneous apoptosis of dendritic cells is efficiently inhibited by TRAP (CD40-ligand) and TNF-alpha, but strongly enhanced by interleukin-10. Eur J Immunol 25: 1943-1950, 1995.

27. Hodge DR, Hurt EM and Farrar WL: The role of IL-6 and STAT3 in inflammation and cancer. Eur J Cancer 41: 2502-2512, 2005.

28. Cirone M, Di Renzo L, Lotti LV, Conte V, Trivedi P, Santarelli R, Gonnella R, Frati L and Faggioni A: Primary effusion lymphoma cell death induced by bortezomib and AG 490 activates dendritic cells through CD91. PLoS One 7: e31732, 2012.

(i) $:$ This work is licensed under a Creative Commons Attribution-NonCommercial-NoDerivatives 4.0 International (CC BY-NC-ND 4.0) License. 